\title{
GASTRECTOMIA EM PACIENTES IDOSOS - ANÁLISE DOS FATORES RELACIONADOS A COMPLICAÇÕES E MORTALIDADE
}

\section{GASTRECTOMY IN ELDERLY PATIENTS - EVALUATION OF THE FACTORS RELATED TO COMPLICATIONS AND MORTALITY}

\author{
Marcelo Garcia Toneto, ACBC-RS ${ }^{1}$; Luís Fernando Moreira, TCBC-RS²; \\ Emílio Jeckel Neto ${ }^{3}$; Hamilton Petry de Souza, TCBC-RS ${ }^{4}$
}

\begin{abstract}
RESUMO: Objetivo: Verificar as variáveis que influenciam os resultados pós-operatórios no tratamento do adenocarcinoma gástrico em pacientes idosos. Método: Foi realizada uma coorte histórica onde as principais variáveis em estudo foram: idade, localização do tumor, estadiamento, doenças associadas, complicações do procedimento cirúrgico e mortalidade operatória. Os pacientes foram separados em dois grupos em relação à idade [Grupo I ( $\leq 65$ anos) e Grupo II (> 65 anos)], de maneira que os fatores associados com maior mortalidade fossem analisados de forma independente. Resultados: Foram avaliados 160 pacientes submetidos à ressecção gástrica. A média de idade foi 60,7 anos. Presença de doenças associadas, classificação ASA III ou IV e mortalidade operatória foram associados com indivíduos mais velhos. Não houve diferença entre os grupos em relação à localização do tumor no estômago, ressecção empregada, estágio dos tumores e complicações pós-operatórias. A mortalidade operatória foi $6,1 \%$ no Grupo I e 12,9\% no Grupo II. O principal fator de risco para o óbito na análise univariada foi a presença de doenças associadas ( $\mathrm{p}<0,03$ ). $\mathrm{Na}$ análise multivariada, o único fator de risco significativo para maior mortalidade foi classificação ASA mais avançada. Conclusão: Os idosos operados por adenocarcinoma gástrico apresentam fator de risco mais acentuado de morte pós-operatória. Contudo, a idade cronológica não pode ser definida como um fator determinante, e sim circunstâncias de doenças associadas e condições fisiológicas que os acompanham nessa faixa etária (Rev. Col. Bras. Cir. 2004; 31(6): 373-379).
\end{abstract}

Descritores: Gastrectomia; Neoplasia gástrica; Estômago; Complicações pós-operatórias; Mortalidade; Idoso.

\section{INTRODUÇÃO}

O aumento da expectativa de vida, devido à diminuição das taxas de mortalidade e intervenções em saúde pública, fez crescer a proporção de pacientes nas faixas mais altas de idade ${ }^{1}$. Não existe um critério cientificamente estabelecido para definir o termo idoso, o qual é utilizado de maneira arbitrária na literatura ${ }^{2}$. À medida que o indivíduo envelhece, aumenta a chance de ocorrer e desenvolver-se algum tipo de câncer, uma vez que mais da metade de todos os casos de tumores malignos são diagnosticados em pessoas com mais de 65 anos de idade ${ }^{3,4}$. Exemplo é a proporção de pacientes acima de 70 anos com diagnóstico de adenocarcinoma gástrico que vem aumentando nos últimos anos ${ }^{5}$. Este tumor é infreqüente em pessoas com idade abaixo de 40 anos $^{6-9}$, sendo a prevalência e a mortalidade significativamente maiores entre indivíduos do sexo masculino com mais de 60 anos de idade $^{5,9-11}$.

O processo de envelhecimento envolve praticamente todos os órgãos, com alterações que podem afetar negativamente a resposta de pacientes idosos submetidos a procedimentos cirúrgicos ${ }^{12}$. Esses indivíduos apresentam uma taxa de morbi-mortalidade maior no período perianestésico em relação aos mais jovens. Contudo, a idade isoladamente pode não ser o fator mais importante a contribuir para esses maus resultados. A presença de problemas cardíacos, respiratórios, imunes, nutricionais e renais pode ser mais importante do que a idade cronológica no resultado final do tratamento $^{13,14}$.

Quando comparados aos pacientes jovens, as taxas de mortalidade e complicações operatórias são maiores em pacientes idosos submetidos à ressecção gástrica. Todavia, permanece controverso se esses pacientes têm uma evolução menos favorável devido à idade ou às condições concomitantes, tais como uma reserva fisiológica limitada, doenças coexistentes ou uma agressividade maior da doença $^{15}$. O objetivo deste estudo é analisar os fatores que influenciam as complicações e mortalidade pós-operatórios da gastrectomia para o tratamento do adenocarcinoma gástrico em pacientes idosos.

\section{MÉTODO}

Foram estudados todos os pacientes submetidos à ressecção cirúrgica por tumores malignos de estômago no Serviço de Cirurgia Geral do Hospital São Lucas da PUCRS, no período de janeiro de 1993 a dezembro de 1998. Foram excluídos do estudo todos os casos com tipo histológico que

1. $\quad$ Professor do Departamento de Cirurgia da FAMED-PUCRS.

2. Professor Adjunto do Programa de Pós-Graduação em Medicina: Cirurgia, Faculdade de Medicina da Universidade Federal do Rio Grande do Sul; Doutor em Cirurgia ( Okayama University - Japão)

3. Doutor em Medicina (Aichi University - Japão); Professor Titular da Faculdade de Biociências - PUCRS

4. Professor Adjunto e Coordenador do Depto Cirurgia da FAMED-PUCRS; Chefe do Serviço de Cirurgia Geral e do Aparelho Digestivo do Hospital São Lucas da PUCRS; Doutor em Cirurgia - UNICAMP. 
não o adenocarcinoma gástrico, pacientes submetidos a procedimentos paliativos que não a ressecção e pacientes submetidos previamente a tratamento rádio e ou quimioterápico.

Trata-se de uma coorte histórica onde as principais variáveis em estudo foram: idade, sexo, localização do tumor no estômago, ressecção de outros órgãos, estadiamento, procedimento cirúrgico realizado, presença de doenças associadas, complicações associadas ao procedimento cirúrgico, tempo de internação hospitalar e mortalidade operatória.

Para efeito de análise, os pacientes foram separados em dois grupos distintos em relação à idade, utilizando-se como ponto de corte um valor que permitisse uma distribuição semelhante no número de óbitos entre os dois grupos. Dessa maneira, os fatores associados com maior mortalidade poderiam ser analisados de forma independente. $\mathrm{O}$ estadiamento utilizado foi a classificação da UICC $1997^{16}$. Quanto ao procedimento cirúrgico, os pacientes foram classificados em três grupos: gastrectomia parcial, total e esofagogastrectomia. Foram avaliadas e descritas as principais doenças concomitantes, e utilizada a classificação de risco validada pela Sociedade Americana de Anestesia (ASA) ${ }^{17}$. Foi definida como complicação qualquer evento pós-operatório que necessitou algum tipo de tratamento adicional aos usualmente utilizados em cirurgias para essa doença. A mortalidade durante o período pós-operatório foi definida como o óbito nos primeiros 30 dias após a cirurgia, independente da alta hospi$\operatorname{talar}^{18}$.

As variáveis quantitativas foram descritas através de média e desvio padrão e as qualitativas com percentuais. A comparação dos grupos foi realizada através do teste $t$ de Student (dados quantitativos), pelo teste do $\mathrm{x}^{2} \mathrm{e}$ exato de Fischer (dados qualitativos) e pelo teste U de Mann-Whitney. Na avaliação da força de associação entre o desfecho óbito e os diversos fatores em estudo, utilizou-se o risco relativo (RR) e seu intervalo de confiança de 95\% (IC 95\%). A significância dessa medida de associação também foi determinada pelo teste do $x^{2}$. Para avaliar o papel de potenciais fatores de confusão especificamente na relação entre idade e óbito, utilizou-se uma análise multivariada baseada no modelo de regressão de Cox. Esse modelo de regressão apresentou estimativas ajustadas para o risco relativo entre os diversos fatores em estudo e a ocorrência de óbito. Os dados foram processados e analisados com o auxílio dos programas Excel 97, SPSS para Windows v 6.0, Epiinfo v 6.0 e PEPI v 2.0. Este estudo foi realizado após aprovação do Comitê de Ética em Pesquisa da Pontifícia Universidade Católica do Rio Grande do Sul

\section{RESULTADOS}

No período de janeiro de 1993 a dezembro de 1998, foram incluídas no estudo 160 ressecções por adenocarcinoma gástrico pelo Serviço de Cirurgia do Hospital São Lucas da PUCRS. A idade variou entre 19 e 91 anos, tendo média (dp) $60,73( \pm 13,86)$ anos. Aidade calculada de 65 anos serviu como ponto de corte para permitir distribuição semelhante no número de óbitos entre os dois grupos.

Os pacientes foram então divididos em dois grupos, 98 pacientes com 65 anos ou menos (Grupo I), e 62 com mais
Tabela 1 - Comparação da classificação ASA entre os dois grupos.

\begin{tabular}{lccr}
\hline Classe & $\begin{array}{c}\text { Grupo I } \\
\mathbf{n}(\boldsymbol{\%})\end{array}$ & $\begin{array}{c}\text { Grupo II } \\
\mathbf{n}(\boldsymbol{\%})\end{array}$ & p \\
\hline ASA I & $26(26,53)$ & 0 & $<0,001$ \\
ASA II & $42(42,85)$ & $22(35,48)$ & 0,450 \\
ASA III & $20(20,40)$ & $29(46,77)$ & $<0,001$ \\
ASA IV & $10(10,20)$ & $11(17,74)$ & 0,259 \\
Total & 98 & 62 & \\
\hline
\end{tabular}

Tabela 2 - Doenças associadas existentes no pré-operatório nos dois grupos.

\begin{tabular}{lccr}
\hline & $\begin{array}{c}\text { GrupoI } \\
\mathbf{n}(\boldsymbol{\%})\end{array}$ & $\begin{array}{c}\text { GrupoII } \\
\mathbf{n}(\boldsymbol{\%})\end{array}$ & Total \\
\hline $\begin{array}{l}\text { Hipertensão arterial } \\
\text { sistêmica }\end{array}$ & $17(17,34)$ & $16(25,80)$ & 33 \\
DPOC* $^{*}$ & $6(6,12)$ & $10(16,12)$ & 16 \\
Cardiopatia isquêmica & $3(3,06)$ & $8(12,09)$ & 11 \\
Alterações cardíacas & $4(4,08)$ & $7(1,61)$ & 11 \\
Diabete melito tipo II & $1(1,02)$ & $3(4,83)$ & 4 \\
Cirrose & $2(2,04)$ & 0 & 2 \\
Outros $_{\text {Total }^{*}}^{12(12,24)}$ & $13(20,96)$ & 25 \\
\hline
\end{tabular}

*DPOC-Doença pulmonar obstrutiva crônica.

** A soma dos valores é maior do que o resultado nos dois grupos devido ao fato de muitos pacientes possuírem mais de uma doença.

de 65 anos (Grupo II). Não houve diferença estatisticamente significativa em relação ao sexo entre os dois grupos. O sexo predominante foi o masculino com $112(70 \%)$ pacientes.

Quanto à classificação da categoria ASA realizada no pré-operatório, $68(69,38 \%)$ pacientes do Grupo I foram classificados como ASA I ou II, ao contrário do Grupo II em que $40(64,51 \%)$ foram classificados com ASA III ou IV (Tabela 1). Setenta e quatro $(46,25 \%)$ pacientes apresentaram doenças associadas, sendo $35(35,71 \%)$ no Grupo I e $39(62,90 \%)$ no Grupo II ( $\mathrm{p}<0,001)$. As principais doenças associadas estão listadas na Tabela 2.

A localização anatômica dos tumores, o tipo de ressecção empregado, a ressecção de outros órgãos e o estadiamento TNM foram semelhantes nos dois grupos.

Cinqüenta e sete $(35,62 \%)$ pacientes apresentaram complicações no período pós-operatório. No Grupo I aconteceram complicações pós-operatórias em $38(38,77 \%)$ casos e no Grupo II em 19 (30,64\%) ( $\mathrm{p}=0,376)$. As complicações mais freqüentes em ambos os grupos foram as respiratórias. A complicação mais grave e responsável pelo maior número de mortes foi a deiscência de sutura das anastomoses ou do coto duodenal (Tabela 3).

A mortalidade geral foi de $14(8,75 \%)$ pacientes, sendo seis $(6,12 \%)$ pacientes no Grupo I e oito $(12,90 \%)$ no Grupo II $(p=0,233)$. As principais complicações que ocasionaram mortes e as características dos pacientes que morreram estão na Tabela 4. 
Tabela 3 - Complicações gerais.

\begin{tabular}{lcrr}
\hline Complicação & $\begin{array}{c}\text { GrupoI } \\
\text { n (\%) }\end{array}$ & $\begin{array}{c}\text { GrupoII } \\
\text { n (\%) }\end{array}$ & Total \\
\hline Complicações respiratórias & $18(18,36)$ & $15(24,19)$ & 33 \\
Deiscência/fístula & $13(13,26)$ & $7(11,29)$ & 20 \\
Infecção ferida operatória & $4(4,08)$ & $3(4,83)$ & 7 \\
Outros & $15(15,3)$ & $11(17,74)$ & 26 \\
Total & $38(38,77)$ & $19(30,64)$ & \\
\hline
\end{tabular}

Oitenta e oito $(55 \%)$ pacientes obtiveram alta hospitalar nos dez primeiros dias após a cirurgia, sendo $60(61,22 \%)$ no Grupo I e 28 (45,16\%) no Grupo II. O tempo de internação variou no Grupo I de cinco a 68 dias, com média (dp) de 13,08 $( \pm 10,74)$ dias e no Grupo II variou de cinco a 48 dias com média de $13,80( \pm 8,42)$ dias, sem no entanto, haver diferença estatisticamente significante $(\mathrm{p}<0,061)$.

A análise independente das variáveis clínicas e de anatomia patológica (Tabela 5) demonstrou que houve diferença significativa para a presença ou não de doenças associadas, para a classificação da ASA e para a mortalidade em relação com a idade. Não houve diferença estatisticamente significativa para o sexo, procedimento cirúrgico realizado, ressecção de outros órgãos, estadiamento ou número de complicações.

A Tabela 6 demonstra o estudo das variáveis em relação aos óbitos ocorridos, nas quais o fator estatisticamente significativo mais importante foi a presença de doenças associadas. Quando se avalia o ponto de corte da idade em 65 anos, o risco relativo de morte é maior para os pacientes acima dessa idade, apesar de não apresentar um valor estatisticamente significativo. Os pacientes ASA III e IV apresentaram um risco relativo maior do que os ASA I e II, porém com um nível de significância limítrofe, da mesma forma que os pacientes com estágios III e IV apresentaram um risco relativo maior de mortalidade.
A Tabela 7 demonstra o resultado da análise multivariada, analisada pelo modelo de Cox, no qual a classificação ASA III e IV foi a variável que apresentou o nível de significância mais importante em relação aos óbitos ocorridos. Idade maior que 65 anos, gastrectomia total ou esofagogastrectomia, e estágio III ou IV não mostraram significância estatística, porém com risco relativo de morte maior.

\section{DISCUSSÃO}

As publicações sobre indivíduos com mais idade que são submetidos a procedimentos cirúrgicos por câncer, muitas vezes são de difícil avaliação para comparação, devido à imprecisão do termo "idoso", o qual é utilizado de maneira arbitrária pelos diversos autores ${ }^{5,9,19-22}$. As faixas etárias utilizadas variam entre 60 e 80 anos e muitos relatos não descrevem claramente as características clínicas e patológicas dos pacientes, necessárias para se realizar as avaliações adequadas. A mortalidade operatória após diversos procedimentos cirúrgicos é maior nas pessoas com mais idade ${ }^{10}$. Contudo, diversos autores concluem que a causa desse aumento não deve ser creditada apenas à idade, mas às complexas alterações que ocorrem gradativamente com o envelhecimento e que ocorrem de maneira e velocidade diferentes entre os vários indivíduos ${ }^{5,18,23}$. A idade, na análise univariada, foi fator de significância estatística para mortalidade aumentada em nossa série. Além dos óbitos, as duas variáveis que mostraram relação significativa com a diferença de idade foram a presença de doenças associadas e a classificação ASA. O estadiamento do tumor, o tipo e a extensão da ressecção empregada e o índice complicações não apresentaram diferença estatisticamente significativa.

Neste estudo, o risco aumentado dos pacientes mais idosos ficou evidente na medida em que os pacientes do Grupo I apresentavam classes ASA menos severas em relação aos pacientes do Grupo II. A presença de doenças associadas

Tabela 4 - Idade e características clínico-patológicas dos pacientes que morreram.

\begin{tabular}{lllllll}
\hline & Idade & Local & Estágio & Ressecção & ASA & Complicação principal \\
\hline Grupo I & & & & & & \\
& 43 & corpo & IV & Parcial & II & deiscência \\
& 58 & fundo/cárdia & IIIA & Total & II & deiscência \\
& 63 & coto & IIIA & Total & IV & deiscência \\
& 64 & antro & II & Parcial & III & deiscência \\
& 65 & corpo & IV & Parcial & IV & deiscência \\
Grupo II & 65 & fundo/cárdia & IV & Esofagogastrectomia & IV & deiscência \\
& 67 & corpo & IIIB & Esofagogastrectomia & II & deiscência \\
& 68 & antro & IIIB & Parcial & III & deiscência \\
& 69 & fundo/cárdia & IIIB & Total & III & deiscência \\
& 69 & corpo & IIIB & Total & III & pneumonia \\
& 69 & antro & IV & Parcial & III & acidente vascular encefálico \\
& 72 & antro & IIIB & Parcial & IV & pneumonia \\
& 78 & fundo/cárdia & IV & Total & II & obstrução jejunostomia \\
& 83 & antro & IIIB & Parcial & IV & insuficiência renal aguda \\
\hline
\end{tabular}


Tabela 5 - Resultado da análise univariada das características clínicas em relação com a idade (anos).

\begin{tabular}{|c|c|c|c|c|c|}
\hline & $\mathbf{n}$ & Média de idade & $\pm d p$ & IC $95 \%$ & $\mathbf{p}$ \\
\hline Masculino & 112 & 61,51 & $\pm 13,23$ & $-2,12$ a 7,32 & 0,278 \\
\hline $\begin{array}{l}\text { Feminino } \\
\quad \text { Doenças associadas }\end{array}$ & 48 & 58,91 & $\pm 15,22$ & & \\
\hline Presentes & 74 & 67,18 & $\pm 9,21$ & 8,20 a 15,80 & $<0,001$ \\
\hline Ausentes & 86 & 55,18 & $\pm 14,80$ & & \\
\hline ASA III-IV & 70 & 67,34 & $\pm 10,15$ & 7,93 a 15,55 & $<0,001$ \\
\hline $\begin{array}{l}\text { ASA I-II } \\
\text { Complicações }\end{array}$ & 90 & 55,60 & $\pm 14,22$ & & \\
\hline Presentes & 57 & 59,91 & $\pm 12,99$ & $-5,81$ a 3,24 & 0,577 \\
\hline $\begin{array}{c}\text { Ausentes } \\
\text { Óbito }\end{array}$ & 103 & 61,19 & $\pm 14,36$ & & \\
\hline Presente & 14 & 66,64 & $\pm 9,21$ & 0,76 a 12,17 & 0,028 \\
\hline $\begin{array}{l}\text { Ausente } \\
\text { Ressecção }\end{array}$ & 146 & 60,17 & $\pm 14,12$ & & \\
\hline Total/esofagogastrectomia & 53 & 59,33 & $\pm 12,72$ & $-6,69$ a 2,53 & 0,371 \\
\hline $\begin{array}{l}\text { Parcial } \\
\quad \text { Ressecção de outros ó }\end{array}$ & 107 & 61,42 & $\pm 14,4$ & & \\
\hline $\operatorname{Sim}$ & 64 & 60,31 & $\pm 12,80$ & $-5,141$ a 3,724 & 0,753 \\
\hline Não & 96 & 61,02 & $\pm 14,58$ & & \\
\hline T2-T3-T4 & 147 & 60,91 & $\pm 13,47$ & $-5,69$ a 9,66 & 0,109 \\
\hline $\mathrm{T} 1$ & 13 & 58,92 & $\pm 17,96$ & & \\
\hline N1-N2-N3 & 109 & 61,17 & $\pm 13,03$ & $-3,28$ a 6,02 & 0,562 \\
\hline $\begin{array}{l}\text { N0 } \\
\text { Estágio }\end{array}$ & 51 & 59,80 & $\pm 15,58$ & & \\
\hline III-IV & 111 & 61,72 & $\pm 13,13$ & $-1,47$ a 7,89 & 0,178 \\
\hline I-II & 49 & 58,51 & $\pm 15,29$ & & \\
\hline
\end{tabular}

e a classificação ASA estiveram associadas ao maior risco relativo de morte de maneira significativa, tanto na análise univariada quanto na multivariada.

A localização da lesão no órgão é importante, pois definirá o tipo de ressecção a ser empregado, fator que pode implicar uma maior morbidade e mortalidade associadas ao procedimento ${ }^{24}$. Tumores no terço distal ou os pequenos tumores do terço médio foram submetidos à gastrectomia parcial. Nos tumores grandes do terço médio e nos localizados no terço proximal, a ressecção foi total. Nos casos de tumores do fundo ou cárdia com invasão do esôfago inferior foi realizada esofagogastrectomia. Siewert et a ${ }^{25}$ relatam que a gastrectomia total é realizada em mais de $70 \%$ dos casos, devido ao fato de muitos tumores estarem localizados no terço proximal do órgão. Nesta série, a localização do tumor no estômago foi independente da idade e os pacientes que foram submetidos à gastrectomia total ou esofagogastrectomia apresentaram maior risco relativo de morte quando comparados com aqueles submetidos à gastrectomia parcial, porém sem significância estatística. Mesmo assim, como os resultados em longo prazo mostram níveis de sobrevida em cinco anos semelhantes com os dois procedimentos mas melhor qualidade de vida para a ressecção parcial ${ }^{26}$, os autores acreditam que esta deva ser a opção nos tumores distais, que permitam margem de ressecção adequada.

O estágio do tumor no momento do diagnóstico é o fator mais importante no prognóstico ${ }^{25-27}$. Além da importân- cia no prognóstico, o estágio influencia na morbidade, pois lesões mais avançadas tendem a ser tratadas com procedimentos de maior porte, e portanto, mais sujeitos a complicações. Não houve diferença no estadiamento dos tumores entre os pacientes dos dois grupos, sendo que menos de $10 \%$ dos pacientes no estudo apresentavam tumores limitados às camadas mucosa e submucosa, isto é, precoces e, portanto, com bom prognóstico. A maioria dos pacientes apresentava tumores em estágio IIIB ou IV, o que demonstra o grau muito avançado da doença no momento do diagnóstico em nosso meio. Esses dados são semelhantes aos encontrados em outros estudos em nosso paí ${ }^{30}$ e em outros países do mundo ocidental ${ }^{29,30}$. A idade não apresentou influência no estadiamento dos pacientes da amostra estudada, semelhante aos dados dos grupos de Kitamura ${ }^{8}$ e de Theurer ${ }^{7}$.

Pires $e t a l^{20}$, em estudo prospectivo de 193 doentes, não mostraram diferença significativa na incidência de complicações entre pacientes jovens e idosos, lembrando a importância de um trabalho em equipe multidisciplinar para uma avaliação adequada no pré-operatório. A complicação mais comum tanto nos pacientes jovens quanto no grupo de mais idade neste trabalho foi a infecção respiratória, com dados semelhantes aos descritos por Saario ${ }^{31}$ e Bittner ${ }^{21}$. A deiscência anastomótica ou do coto duodenal foram as complicações mais graves, sendo responsáveis pelo óbito em nove $(64,28 \%)$ dos 14 pacientes que morreram. Todos os pacientes que morreram no grupo I tiveram como causa a deiscência, dados se- 
Tabela 6 - Resultado da análise univariada das características clínicas em relação aos óbitos.

\begin{tabular}{|c|c|c|c|c|c|c|}
\hline \multicolumn{7}{|c|}{ Óbitos } \\
\hline & $\mathbf{n}$ & $f^{*}$ & $\%$ & $\mathbf{R R}^{* * *}$ & IC $95 \%$ & p \\
\hline \multicolumn{7}{|l|}{ Idade } \\
\hline$>65$ anos & 62 & 8 & 12,9 & 2,10 & $-0,76$ a 5,78 & 0,233 \\
\hline$\leq 65$ anos & 98 & 6 & 6,1 & & & \\
\hline Masculino & 112 & 11 & 9,8 & 1,57 & 0,45 a 5,38 & 0,667 \\
\hline Feminino & 48 & 3 & 6,3 & & & \\
\hline \multicolumn{7}{|l|}{ Doenças associadas } \\
\hline Presentes & 74 & 11 & 14,9 & 4,261 & 1,23 a 14,69 & 0,024 \\
\hline Ausentes & 86 & 3 & 3,5 & & & \\
\hline ASA III-IV & 70 & 10 & 14,3 & 3,21 & 1,05 a 9,81 & 0,057 \\
\hline ASA I-II & 90 & 4 & 4,4 & & & \\
\hline \multicolumn{7}{|l|}{ Gastrectomia } \\
\hline Total/esofagogastrectomia & 53 & 7 & 13,2 & 2,01 & 0,74 a 5,45 & 0,232 \\
\hline Parcial & 107 & 7 & 6,5 & & & \\
\hline \multicolumn{7}{|l|}{ Ressecção outros órgãos } \\
\hline Sim & 64 & 9 & 14,1 & 2,70 & 0,94 a 7,68 & 0,098 \\
\hline Não & 96 & 5 & 5,2 & & & \\
\hline T2-T3-T4 & 147 & 14 & 9,5 & 0,90 & $0,85 \mathrm{a} 0,95$ & 0,614 \\
\hline $\mathrm{T} 1$ & 13 & 0 & 0 & & & \\
\hline N1-N2-N3 & 109 & 12 & 11,0 & 2,8 & 0,65 a 12,08 & 0,228 \\
\hline NO & 51 & 2 & 3,9 & & & \\
\hline \multicolumn{7}{|l|}{ Estágio } \\
\hline III-IV & 111 & 13 & 11,7 & 5,73 & $0.77 \mathrm{a} 42.65$ & 0.067 \\
\hline I-II & 49 & 1 & 2,0 & & & \\
\hline
\end{tabular}

* - Freqüência

** - Risco relativo

$\dagger$ - Intervalo de confiança

melhantes aos encontrados numa análise de 700 casos em trabalho realizado em conjunto em centro americano e russo por Shchepotin et al ${ }^{32}$ e na revisão de $\mathrm{McCulloch}^{33}$. A faixa etária não teve relação com o índice de complicações em nosso estudo, ao contrário da mortalidade, o que sugere que os indivíduos de mais idade não suportam as intercorrências pósoperatórias como os mais jovens. As causas de morte dos indivíduos mais velhos foram devidas a problemas clínicos intervenientes, ao contrário dos mais jovens em que a causa foi sempre a complicação abdominal relacionada com o procedimento cirúrgico, resultados semelhantes aos descritos no grupo de Moreno-Gonzalez?.

A mortalidade cirúrgica dos pacientes submetidos à ressecção do câncer gástrico varia muito conforme os diversos grupos que tratam dessa doença ${ }^{21,22,26,34,35}$. Os fatores de

Tabela 7 - Análise multivariada para os óbitos.

\begin{tabular}{|c|c|c|c|}
\hline & $\mathbf{R R}$ & IC $95 \%$ & $\mathbf{p}$ \\
\hline Idade $>65$ anos & 2,23 & 0,60 a 8,19 & 0,228 \\
\hline ASA III-IV & 3,31 & 0,82 a 13,36 & 0,092 \\
\hline Gastrectomia total & 2,48 & 0,54 a 11,23 & 0,237 \\
\hline $\begin{array}{l}\text { Ressecção de } \\
\text { outros órgãos }\end{array}$ & 0,80 & 0,17 a 3,56 & 0,769 \\
\hline Estágio III ou IV & 1,92 & 0,22 a 16,45 & 0,551 \\
\hline
\end{tabular}

risco para mortalidade operatória são a presença de doenças concomitantes, metástases linfonodais, tamanho do tumor, experiência do grupo de cirurgiões e a idade ${ }^{11}$. A alta mortalidade encontrada nesta série, apesar de similar a estudos recentes $^{36}$, provavelmente se deve à inclusão de cirurgias paliativas, em doentes em condições de estado fisiológico préoperatório precárias e em estágios tumorais avançados. Salienta-se que não houve mortalidade entre os pacientes com tumor precoce e, apenas um paciente em estágio II, com 64 anos, apresentou fístula do coto duodenal que desencadeou sepse, levando ao óbito.

Diversos estudos demonstram que a idade é fator de pior prognóstico em relação a um maior número de complicações e óbitos, quando os dados são avaliados pelo método de análise univariada ${ }^{22,23,30}$. Quando esses dados são submetidos a análises de regressão multivariada, outros fatores demonstram maior importância do que a idade, tais como a presença concomitante de outras doenças ou um número maior de procedimentos cirúrgicos realizados em caráter de urgência ${ }^{15}$. Quanto à mortalidade dos pacientes em estágio III e IV, o risco relativo de morte nesses pacientes foi mais do que cinco vezes superior aos que estavam em estágios iniciais na análise univariada, e cerca de duas vezes maior na multivariada, porém sem diferença estatística. Talvez o pequeno número de pacientes nos subgrupos da amostra não tenha permitido definir melhor a relação entre o estágio da doença e a mortalidade cirúrgica. 
Os resultados encontrados neste estudo demonstram que a mortalidade foi maior nos pacientes com mais idade. Os fatores relacionados a esses achados foram à presença de um maior número de doenças associadas e as piores condições de reserva fisiológica neste grupo refletida numa classificação ASA pré-operatória significativamente maior, semelhante aos dados do grupo de Moreno-Gonzalez ${ }^{9}$. Estes resultados sugerem que a ressecção cirúrgica pode ser empregada para o tratamento do câncer gástrico em pacientes idosos a não ser que o tumor esteja em estágio muito avançado ou que as condições fisiológicas do paciente não permitam. Um au- mento no número de pacientes da amostra, principalmente com tumores mais iniciais, certamente contribuiria para definir melhor a questão da importância do estágio e da extensão da ressecção cirúrgica na mortalidade pós-operatória.

Os resultados deste trabalho sugerem que os pacientes idosos devam ser incluídos em investigações que avaliem a efetividade do tratamento cirúrgico do adenocarcinoma gástrico, uma vez que é o estado físico e biológico do indivíduo e não a idade cronológica, que deve servir como parâmetro para selecionar os pacientes para o procedimento cirúrgico.

\begin{abstract}
Background: The aim of this study was to evaluate the outcome of gastrectomy in elderly patients. Methods: In a historical cohort, variables such as age, site and stage of tumor, associated diseases, morbidity, and mortality were studied. The patients were divided, by age, into two groups and statistical analysis was performed to determine variables related to postoperative complications or death. Results: We studied 160 patients submitted to gastric resection for adenocarcinoma. Mean age of the patients was 60.7 years. Associated diseases, ASA score III or IV, and mortality were significantly higher according to the age. The patients were divided into two groups: those under 65 years (Group I) and those over 65 years (Group II). No statistically significant difference between the groups regarding tumor site and stage, type of resection, and rate of postoperative complications were observed. Hospital mortality was $6.1 \%$ in group I and $12.9 \%$ in group II. Associated illness was the main factor related to death in this series in the univariate analysis $(P<0.03)$. On multivariate analysis, the only significant risk factor was a higher ASA score. Conclusion: Our results suggest that elderly patients with gastric cancer have an increased risk of postoperative death. However, age itself could not be identified as an isolated factor, accompanying illness and physiologic conditions seemed to be more predictive.
\end{abstract}

Key words: Gastrectomy; Gastric Neoplasms; Stomach; Postoperative complications; Mortality; Aged.

\section{REFERÊNCIAS}

1. Keating HJ, Lubin MF - Perioperative responsabilities of the physician/geriatrician. Clin Geriatr Med, 1990, 6(3):459-467.

2. Alexiou C, Beggs D, Salama FD, et al. - Surgery for esophageal cancer in elderly patients: the view from Nottingham. J Thorac Cardiovasc Surg, 1998,116(4):545-553.

3. Pitot HC - Aging and cancer: some general thoughts. J Gerontol, 1989,44(6):5-9.

4. Witten M-Modeling of the aging-cancer interface: some thoughts on a complex biological dynamics. J Gerontol, 1989,44(6):7280.

5. Maehara Y, Oshiro T, Oiwa H, et al. - Gastric carcinoma in patients over 70 years of age. Br J Surg,1995,82(1):102-105.

6. El-Kadre LJ, Rodrigues FCM, Malheiros CA, et al. - Carcinoma gástrico em pacientes com menos de 40 anos de idade. Rev Col Bras Cir,1995,22(3):117-119.

7. Theuer CP, de Virgilio C, Keese G, et al. - Gastric adenocarcinoma in patients of 40 years of age or younger. Am J Surg, 1996,172(5):473-477.

8. Kitamura K, Yamaguchi T, Yamamoto K, et al. Clinicopathological analysis of gastric cancer in young adults. Hepatogastroenterology, 1996, 43(11):1273-1280.

9. Moreno-Gonzalez E, Gomez Gutierrez M, Landa Garcia I, et al. - Câncer gástrico no paciente maior de 65 anos. Estudo comparativo dos diferentes tipos de ressecção. Rev Col Bras Cir, 1988,15(5):301-307.

10. Zenilman ME - Surgery in the elderly. Curr Probl Surg, 1998,35(2):99-179.

11. Siewert JR, Fink U, Sendler A, et al. - Gastric cancer. Curr Probl Surg, 1997,34(11):835-939
12. Evers BM, Townsend CM, Thompson JC - Organ physiology of aging. Surg Clin North Am, 1994,74(1):23-39.

13. Fong Y, Blumgart LH, Fortner JG, et al. - Pancreatic or liver resection for malignancy is safe and effective for the elderly. Ann Surg 1995,222(4):426-437.

14. Gagner M - Valor da avaliação fisiológica pré-operatória no prognóstico de pacientes submetidos a procedimentos de grande porte. Clin Cir Am Norte, 1991,6:1215-1226.

15. Layon AJ, George BE, Hamby B, et al. - Do elderly patients overutilize healthcare resources and benefit less from them than younger patients? A study of patients who underwent craniotomy for treatment of neoplasm. Crit Care Med, 1995,23(5):829-834.

16. Hermanek P - pTNM and residual tumor classifications: problems of assessment and prognostic significance. World J Surg, 1995,19(2):184-190.

17. Cullen DJ, Apolone G, Greenfield S, et al. - ASA Physical Status and age predict morbidity after three surgical procedures. Ann Surg, 1994,220(1):3-9.

18. Schwarz RE, Karpeh MS, Brennan MF - Factors predicting hospitalization after operative treatment for gastric carcinoma in patients older than 70 years. J Am Coll Surg, 1997,184(1):9-15.

19. Hanazaki K, Wakabayashi M, Sodeyama H, et al. - Surgery for gastric cancer in patients older than 80 years of age. Hepatogastroenterology, 1998,45(19):268-275.

20. Pires PW, Bevilacqua RG, Birolini D - Cirurgia do paciente idoso. Doenças associadas e complicações pós-operatórias: Estudo prospectivo em 193 doentes. Rev Col Bras Cir, 1995,21(5):261-265.

21. Bittner R, Butters M, Ulrich M, et al. - Total gastrectomy. Updated operative mortality and long-term survival with particular reference to patients older than 70 years of age. Ann Surg, 1996,224(1):37-42. 
22. Oliveira FJ, Furtado E, Ferrao H, et al. - Total gastrectomy for gastric cancer in elderly patients. Hepatogastroenterology, 1999,46(25):616-619.

23. Seymour DG, Faz FG. A prospective study of elderly general surgical patients: II. Post-operative complications. Age Ageing, 1989,18(5):316-326

24. Longmire WP Jr - A current view of gastric cancer in the US. Ann Surg, 1993,218(5):579-583.

25. Bollschweller E, Boettcher K, Hoelsche AH, et al. - Is the prognosis for Japanese and German patients with gastric cancer really different? Cancer, 1993,71(10):2918-2925.

26. Bozzetti F, Marubini E, Bonfanti G, et al. - Subtotal versus total gastrectomy for gastric cancer: five-year survival rates in a multicenter randomized Italian trial. Italian Gastrointestinal Tumor Study Group. Ann Surg, 1999,230(2):170-178.

27. Wanebo HJ, Kennedy BJ, Chmiel J, et al. - Cancer of the stomach. A patient care study by the American College of Surgeons. Ann Surg, 1993,218(5):583-592

28. Hundahl SA, Menck HR, Mansour EG, et al. - The National Cancer Data Base report on gastric carcinoma. Cancer, 1997,80(12):2333-2341.

29. Bringaze WL, Chappuis CW, Correa P, et al. - Early gastric cancer. 21- year experience. Ann Surg, 1986,204(2):103-107.

30. Bevilaqua RG, Alcântara PS, Zanvettor PH, et al. - Fatores prognósticos das complicações pós-operatórias nas ressecções por câncer gástrico. Rev Col Bras Cir, 1996,23(1):51-56.
31. Saario I, Schroder T, Tolppanen EM, et al. - Total gastrectomy with esophagojejunostomy. Analysis of 100 consecutive patients. Am J Surg, 1986,151(2):244-248.

32. Shchepotin IB, Evans SR, Chorny VA, et al. - Postoperative complications requiring relaparotomies after 700 gastrectomies performed for gastric cancer. Am J Surg, 1996,171(2):270-273.

33. McCulloch $\mathrm{P}$ - Should general surgeons treat gastric carcinoma? An audit of practice and results, 1980-1985. Br J Surg, 1994,81(3):417-420.

34. Japanese Research Society for Gastric Cancer. Treatment results of gastric carcinoma in Japan: $39^{\text {th }}$ report of nationwide registry in 1979-82. Tokio: National Cancer Center Press, 1995.

35. Crookes PF, Incarbone R, Peters JH, et al. - A selective therapeutic approach to gastric cancer in a large public hospital. Am J Surg, 1995,170(6):602-605.

36. Callahan MA, Christos PJ, Gold HT, et al. - Influence of surgical subspecialty training on in-hospital mortality for gastrectomy and colectomy patients. Ann Surg, 2003,238(4):629-639.

Endereço para correspondência:

Marcelo Garcia Toneto

Centro Clínico da PUC

Av Ipiranga, 6690 - Conj 302

CEP 90610-000 - . Porto Alegre, RS

Fone: 33360890 / 33395796.

E-mail: mtoneto@terra.com.br 